Publ. RIMS, Kyoto Univ.

20 (1984), 893-896

\title{
On the $\operatorname{Mod} p$ Decomposition of $Q\left(C P^{\infty}\right)$
}

\author{
Dedicated to Professor Nobuo Shimada on his 60th birthday
}

By

\section{Akira KonO*:}

\section{§1. Introduction}

Let $j: C P^{\infty} \rightarrow B U$ be the natural inclusion and $\xi: Q(B U) \rightarrow B U$ be the structure map of the infinite loop space structure defined by the Bott periodicity theorem, where

$$
Q(\mathrm{Y})=\underset{n}{\operatorname{Colim}} \Omega^{n} \Sigma^{n} X
$$

for a pointed space $X$. In $[5]$ Segal defined a map $\lambda: Q \mathbb{C}^{C} P^{\cdots} \rightarrow B L^{\prime}$ by the composition

$$
Q\left(C P^{\circ 0}\right) \stackrel{Q(j)}{\longrightarrow} Q(B U) \stackrel{\xi}{\longrightarrow} B L^{-}
$$

and showed that there exists a map $s: B U \rightarrow Q\left(C P^{\sim}\right)$ such that $\lambda \cdot s \simeq 1$. As a corollary of the above fact he showed that there is a space $F$ satisfying $Q\left(C^{\circ}\right.$; $\simeq B U \times F$ and $\pi_{*}(F)$ is a finite abelian group for any *.

Let $p$ be a rational prime. In [1] Adams showed cinat there exist infinite loop spaces $G_{1}, \cdots, G_{1^{\prime-1}}$ such that

$$
B L_{(p)}^{r} \simeq \prod_{k=1}^{p-1} G_{k}
$$

where $B U_{(p)}$ denotes the localization at $p$ (for details see $\$ 2$. On the other hand by Mimura, Nishida and Toda [4], there exist $I_{1}, \cdots, I_{2,-1}$ such that

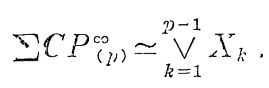

Then

$$
Q\left(\mathbb{C} P_{(p)}^{\infty}\right) \simeq Q Q\left(\Sigma C P_{(p)}^{\infty}\right) \simeq \Omega Q\left(\bigvee_{k=1}^{p-1} X_{i}\right) \simeq \prod_{i=1}^{i} \prod_{i=1}^{p-1} Q Q_{1} I_{i}
$$

(see $\$ 3$ ). The purpose of this paper is to show

Theorem 1.1. There cxists $F_{i_{0}}$ such that

$$
\Omega Q\left(Y_{k_{0}}\right) \simeq G_{k_{0}} \backslash F_{k_{11}}
$$

and $\pi_{*}\left(F_{k_{0}}\right)$ is finite for each $k_{0}\left(1 \leqq k_{0} \leqq p-1\right)$.

Communicated by N. Shimada, September 30, 1983.

"Dapartment of Mathematics. Kyoto Lniversity, Kyoto 600. Japar 
Noting that $Q\left(X_{(p)}\right) \simeq Q(X)_{(p)}$ by Lemma 3.1, we have

$$
Q\left(C P^{\infty}\right)_{(p)} \simeq\left(\prod_{k=1}^{p-1} G_{k}\right) \times\left(\prod_{k=1}^{p-1} F_{k}\right) .
$$

Let $n$ be a positive integer which divides $p-1$. In $[6]$, Sullivan showed that $S_{(p)}^{2 n-1}$ is an associative $H$-space. In [3], McGibbon showed that

$$
\Sigma B S_{(p)}^{2 n-1} \simeq \underset{k=1}{(p-1) / n} X_{n k}
$$

(the definition of $X_{k}$ in [3] is the same as that in [4]). Thus we have

Corollary 1.2. If $n$ is a positive integer which divides $p-1$, then

$$
Q\left(B S_{(p)}^{2 n-1}\right) \simeq\left(\prod_{k=1}^{(p-1) / n} G_{n k}\right) \times\left(\prod_{k=1}^{(p-1) / n} F_{n k}\right) \text {. }
$$

\section{$\S 2$. Mod $p$ Decomposition of the Complex $K$-Theory}

Let $p$ be a rational prime and $K^{*}()_{(p)}$ be the complex $K$-theory localized at p. The following is due to Adams (see Lecture 4 of [1] and $\S 9$ of [2]) :

Tneorem 2.1. There exist (generalized) cohomology theories $E_{1}^{*}(), \cdots, E_{p-1}^{*}()$ satisfying

(1) as a cohomology theory $K^{*}()_{(p)}=E_{1}^{*}() \oplus \cdots \oplus E_{p-1}^{*}()$, and

(2) $E_{k}^{a}(p t)= \begin{cases}Z_{(p)} & \text { if }-a \equiv 2 k \bmod 2(p-1) \\ 0 & \text { otherwise }\end{cases}$ $(1 \leqq k \leqq p-1)$.

Let $e_{k}^{*}()$ be the associated connective cohomology theory of $E_{k}^{*}()$ and $G_{k}$ be an infinite loop space which represents $e_{k}^{*}()$. As a corollary of the above theorem, we have the following:

Corollary 2.2. There exists a homotopy equivalence

$$
B U_{(p)} \simeq \prod_{k=1}^{p-1} G_{k}
$$

and the homotopy groups of $G_{k}$ are given by

$$
\pi_{a}\left(G_{k}\right)= \begin{cases}Z_{(p)} & \text { if } a \equiv 2 k \bmod 2(p-1) \text { and } a>0, \\ 0 & \text { otherwise. }\end{cases}
$$

The following is due to Mimura, Nishida and Toda (see [4]) :

Lemma 2.3. There exist $X_{1}, \cdots, X_{p-1}$ such that

(1) $\Sigma C P_{(p)}^{\infty} \simeq \bigvee_{k=1}^{p-1} X_{k}$ 
and

(2) $\tilde{H}_{a}\left(X_{k}\right)= \begin{cases}Z_{(p)} & \text { if } a-1 \equiv 2 k \bmod 2(p-1) \text { and } a>1, \\ 0 & \text { otherwise. }\end{cases}$

\section{§3. Proof of Theorem 1.1}

Let $X$ be a connected, simply connected $C W$ complex. Then $\Sigma Y_{(p)} \simeq \Sigma I_{(p)}$ and $(\Omega X)_{(p)} \simeq \Omega Y_{(p)}$ (cf. [4]). The following is easily prored:

Lemma 3.1. For any pointed $C W$ complex $\left.X, Q(X)_{(\mu)} \simeq Q I_{(n)}\right)$.

The homotopy equivalence in Lemma 2.3 induces homotopy equivalences

$$
Q\left(C P_{(p)}^{\infty}\right) \simeq \Omega Q\left(\Sigma C P_{(p)}^{\infty}\right) \simeq \prod_{k=1}^{p-1} \Omega Q\left(X_{k}\right) .
$$

Let $j_{k}: \Omega Q\left(X_{k}^{\prime}\right) \rightarrow Q\left(C P_{(p)}^{\infty}\right)$ and $j_{k}^{\prime}: G_{k} \rightarrow B U_{(p)}$ be the natural inclusions and $q_{k}: Q\left(C P_{(p)}^{\infty}\right) \rightarrow \Omega Q\left(X_{k}\right)$ and $q_{k}^{\prime}: B U_{(p)} \rightarrow G_{k}$ be the natural projections $(1 \leqq k \leqq p-1)$. Put

and

$$
\lambda_{k}=q_{k}^{\prime} \circ \lambda_{(p)}: Q\left(C P_{(p)}^{\infty}\right) \rightarrow B U_{(p)} \rightarrow G_{k}
$$

Then we have

$$
s_{k}=S_{(p)} \circ j_{k}^{\prime}: G_{k} \rightarrow B U_{(p)} \rightarrow Q\left(C P_{(p)}^{\infty}\right)
$$

$$
\lambda_{k} \circ S_{k}=q_{k}^{\prime} \circ \lambda_{(p)} \circ S_{(p)} \circ j_{k}^{\prime} \simeq q_{k}^{\prime} \circ j_{k}^{\prime} \simeq 1_{G_{k}} .
$$

Now to prove Theorem 1.1, we need only show the following see ${ }_{-5}$ ]):

Theorem 3.2. For each $k_{0}$, the composition

is a homotopy equivalence.

$$
\left(\lambda_{k_{0}} \circ j_{k_{0}}\right) \circ\left(q_{k_{0}} \circ S_{k_{0}}\right)
$$

The following is proved by a standard argument (cf. [5?):

Lemma 3.3. The homotopy groups of $\Omega Q\left(X_{k}\right)$ are given $b_{-1}$

$$
\pi_{a}\left(Q Q\left(X_{k}\right)\right)=\left\{\begin{array}{l}
Z_{(p)} \oplus p \text {-torsion if } a \equiv 2 k \bmod 2(p-1) \text { and } a \backslash 0, \\
p \text {-torsion otherwise. }
\end{array}\right.
$$

To prove Theorem 3.2, we need the following algebraic lemma:

Lemma 3.4. Let $R$ be a (commutative) ring (with unt $y$, $\dot{I}: A \rightarrow B$ and $s: B \rightarrow A$ be an $R$-module homomorphism such that $f_{\circ S}=1_{B}$. Sitnose that there is a direct sum decomposition $A=A_{1} \oplus \cdots \oplus A_{n}$ of $R$-modules uith the projection $p_{k}: A \rightarrow A_{k}$ and the inclusion $i_{k}: A_{k} \rightarrow 4 \quad(1 \leqq k \leqq n)$. If $B$ is a free R-module and there is an integer $k_{0}\left(1 \leqq k_{n} \leqq n\right)$ such that $A_{k}$ is a torsion $R$-mudule for cach 
$k \div k_{v}$, then $f_{k} \circ s_{k}=\bar{o}_{k, k_{u}} \circ 1_{B}$, where $f_{k}=f \circ i_{k}$ and $s_{k}=p_{k} \circ s_{\text {. }}$

Proof of Lemma 3.4. If $k \neq k_{0}$, then $f_{k}=0$, since $A_{k}$ is a torsion $R$-module and $B$ is a free $R$-module. Therefore $f_{k} \circ s_{k}=0$ if $k \neq k_{0}$ and

$$
f_{k_{0}} \circ s_{\dot{k}_{0}}=\sum_{k=1}^{n} f_{k} \circ s_{k}=\sum_{k=1}^{n} f_{\circ} i_{k} \circ p_{k} \circ s=f \circ\left(\sum_{k=1}^{n} i_{k} \circ p_{k}\right) \circ=f \circ s=1_{B} .
$$

Proof of Theorem 3.2. Fix an integer $k_{0}\left(1 \leqq k_{0} \leqq p-1\right)$. Let $a$ be a positive integer such that $a \equiv 2 k_{0} \bmod 2(p-1)$. Then to prove Theorem 3.2 , we need only show

$$
L i=\lambda_{k_{0}} \circ j_{k_{0}^{*} \circ}^{*} q_{k_{0}^{*} \circ}^{*} S_{k_{0}^{*}}: \pi_{a}\left(G_{k_{0}}\right) \rightarrow \pi_{a}\left(G_{k_{0}}\right)
$$

is an isomorphism. Put $R=Z_{(p)}, A=\pi_{a}\left(Q\left(\mathbb{C} P_{(p)}^{\infty}\right)\right), A_{k}=\pi_{a}\left(\Omega Q\left(X_{k}\right)\right), B=\pi_{a}\left(G_{k_{0}}\right)$, $f=\lambda_{k_{0}^{*}}$ and $s=s_{k_{j}}$. Then clearly $j_{k^{*}}=i_{k}$ and $q_{k^{*}}=p_{k}$. Since $\lambda_{k_{0}^{*} \circ{ }^{*}}=1_{B}$ by (*), $A_{k}$ is a $p$-torsion group if $k \neq k_{0}$ by Lemma 3.3 and $B$ is a free $Z_{(p)}$-module by Corollary 2.2, $\quad i=1_{z}$ by Lemma 3.4.

\section{References}

[1] Adams. J.F., Lectures on generalized cohomology, Lecture Notes in Math., 99, 1138.

[2:- Araki, S., Trpical formal groups in complex cobordism and K-theory, Lectures in Math., Deparment of Math., Kyoto Univ., 6, 1973.

[3.- McGibbon, C.A.. Stable properties of rank 1 loop structure, Topology, 20 (1981), 109-118.

[4] Mimura, M., Nishida, G. and Toda, H., Localization of CW complex and its applications, J. Math. Soc. Japan, 23 (1971), 593-624.

[5] Segal, G.. The stable homotopy of complex projective space, Quart. J. Math. Oxford (2), $2 \pm$ (1973), 1-5.

[o] Sullivan, D., Geometric topology: Part I, Localization, Periodicity and Galois symmetry, MIT Ninies. 1970. 